\title{
Relation between flux formation and pairing in doped antiferromagnets
}

\author{
P. Wróbel ${ }^{1}$ and R. Eder ${ }^{2}$ \\ ${ }^{1}$ Institute for Low Temperature and Structure Research, P. 0. Box 1410, 50-950 Wroctaw 2, Poland \\ 2 Institut für Theoretische Physik, Universität Würzburg, Am Hubland, 97074 Würzburg, Germany
}

(October 26, 2018)

\begin{abstract}
We demonstrate that patterns formed by the current-current correlation function are landmarks which indicate that spin bipolarons form in doped antiferromagnets. Holes which constitute a spin bipolaron reside at opposite ends of a line (string) formed by the defects in the antiferromagnetic spin background. The string is relatively highly mobile, because the motion of a hole at its end does not raise extensively the number of defects, provided that the hole at the other end of the line follows along the same track. Appropriate coherent combinations of string states realize some irreducible representations of the point group $C_{4 v}$. Creep of strings favors $d$ - and $p$-wave states. Some more subtle processes decide the symmetry of pairing. The pattern of the current correlation function, that defines the structure of flux, emerges from motion of holes at string ends and coherence factors with which string states appear in the wave function of the bound state. Condensation of bipolarons and phase coherence between them puts to infinity the correlation length of the current correlation function and establishes the flux in the system.
\end{abstract}

PACS numbers: 74.25.Jb, 71.10.Fd, 71.27.+a

\section{INTRODUCTION}

Flux phase appeared in the mean-field approaches to undoped and doped antiferromagnets described by the $t$ - $J$ modell. The physical meaning of that phase and its relation with pairing was not clear. In some announcements 1 目 the competition or coexistence between superconductivity and the flux phase have been discussed in the framework of the mean filed approach and the $1 / N$ expansion. Some researchers tend to understand the spin gap phasein doped antiferromagnets as the staggered flux phase $\mathrm{d}$. Bther believe that pairing grows out of the $\pi$-flux phase 3 or that pairs are formed by holes circulating in opposite directions 10 . In this paper we will present a different point of view that the pattern of currents and pairing originate in formation of spin bipolarons of a particular form. We will give some mathematical arguments, based on understanding of pairing in weakly doped antiferromagnets, in support of that idea.

Recently, Ivanov, Lee and Went0 reported staggeredvorticity correlations of the current-current correlation function $\left\langle j_{i j} j_{k l}\right\rangle(\mathrm{CCCF})$ in the $d$-wave variational functions for a weakly doped antiferromagnet, where $j_{i j}$ denotes the current flowing on the bond $\langle i, j\rangle$. Similar pattern of the CCCF was found for a bound state of two holes in an exact diagonalization performed by Leung 11 . Since the mechanism of hole binding in weakly doped antiferromagnets is relatively well understood 12 , it is a tempting task to resolve whether there exist a deep relation between binding and the formation of flux patterns.

Fast motion, with the rate $\sim t$, of a hole created at some site in the Nèel stateforms defects in the original arrangement of spins 13,4 . These defects, that are spins which have been turned upside down and may also be called magnons, lie on the track of the hole. Strings pinned to a central site form a potential well for a hole which confines its motion in the vicinity of this site. Much slower processes related to the inversion of antiparallel spins at nearest neighbor sites which occur at the rate $\sim J$ shorten strings and give rise to coherent propagation of the hole. Since the separation of energy scales for hopping of a hole and annihilation of defects (magnons) is pronounced it is plausible to introduce the notion of a spin polaron. We may define in this context a spin polaron as a solution of the Schrödinger equation for a particle in the potential well15. Slower processes are neglected at this stage of analysis. "Orbital" states of polarons created at all possible sites exhaust, in principle, the relevant portion of the Hilbert space. In practice, the calculation may be confined to some low excited states or even to the polaron groundstate. By calculating the matrix elements of the full Hamiltonian in the polaron basis we derive an effective Hamiltonian expressed in the polaron language. Some processes, like oscillations of the hole in the vicinity of a polaron center, are already incorporated into the eigenenergy of polaronic states. The rest either renormalizes the eigenenergies or gives rise to offdiagonal matrix elements in the polaronic Hamiltonian. The latter circumstance occurs in the case of shortening of strings by an appropriate term in the original Hamiltonian. The approach outlined above has some obvious limitations. It is applicable provided that the correlation length of background antiferromagnetic spin correlations is larger than the polaron radius.

We extend now the concept of spin polarons to the description of interaction between holes 16 . Since we are going to apply this construction to the calculation of the current correlation function, we will provide the reader with more details. We define a spin bipolaron $\left|\Psi_{\langle i, j\rangle}\right\rangle$ as a combination of states which may be reached by in- 
dependent hopping of holes created at a pair of nearest neighbor sites $\langle i, j\rangle$,

$$
\left|\Psi_{\langle i, j\rangle}\right\rangle=\sum_{\mathcal{P}_{i}, \mathcal{P}_{j}} \alpha_{\mathcal{P}_{i}, \mathcal{P}_{j}}\left|\mathcal{P}_{i}, \mathcal{P}_{j}\right\rangle
$$

$\mathcal{P}_{i}$ parameterizes the geometry of a path along which the hole has been moving and $\left|\mathcal{P}_{i}, \mathcal{P}_{j}\right\rangle$ is a state which has been created in this way. Due to Fermi statistics, order in which holes have been originally created in $\left|\Psi_{\langle i, j\rangle}\right\rangle$ is relevant. We adopt a convention, according to which a hole is created first at the site $i$ and $i$ belongs to the even sublattice. At this stage of considerations we prohibit, by definition, each hole to follow along the trace left by the accompanying hole. By means of that somewhat artificial restriction we achieve that spin bipolarons are localized and we may proceed as in the case of a single hole. We make a further approximation and neglect all path details including the possibility of path crossing. It is self-evident that the coefficients $\alpha_{\mathcal{P}_{i}, \mathcal{P}_{j}}$ for a bipolaron in the groundstate will depend only on the lengths $\mu, \nu$ of paths $\mathcal{P}_{i}$ and $\mathcal{P}_{j}, \alpha_{\mathcal{P}_{i}, \mathcal{P}_{j}}=\alpha_{\mu, \nu}$. The Schrödinger equation that describes a hole pair in a potential well and defines the spin bipolaron, may be written as

$$
\begin{array}{r}
t\left[\alpha_{\mu-1, \nu}+(z-1) t \alpha_{\mu+1, \nu}+\alpha_{\mu, \nu-1}+(z-1) t \alpha_{\mu, \nu+1}\right] \\
+J\left(4+\mu+\nu-\frac{1}{2} \delta_{\mu, \nu}\right) \alpha_{\mu, \nu}=E_{2} \alpha_{\mu, \nu}
\end{array}
$$

where $\alpha_{\mu, \nu}=0$ for $\mu<0$ or $\nu<0$ and $\mathrm{z}=4$. The form of this equation is easy to understand. The first expression in it is related to the fact that each path may be reached from one shorter path and (z-1) different longer paths by a hop of the hole. The second term counts the number of pairs of nearest neighbor sites, that are not occupied by anti-parallel spins. Every such a "broken bond" raises the energy by $J / 2$ in comparison to the energy of the Néel state.

Formation of bipolarons, and, in particular, strings that connect holes, is an effective way of lowering energy. A compromise between two opposite tendencies to minimize the kinetic energy of holes and to reduce the disturbance of the antiferromagnetic background is reached by means of that process. By shrinking at one end and expanding at the opposite-end, a string may move, while keeping a moderate length 17 . The application of the spinpolaron scenario to binding of holes in doped antiferromagnets gave rise to better than qualitative agreement with results of numerical diagonalization 18 . In particular the hierarchy of states that realize irreducible representations for all momenta and symmetries allowed by the geometry of the $4 \times 4$ cluster have been reproduced 12 . It turns out that creep of strings favors $d$-wave and $p$ wave states. More subtle processes decide the symmetry of the bound state in favor of the $d$-wave. Some modifications of the $t-J$ model, like asymmetry of the magnetic interaction may lead to stability of the $p$-wave state 29 . The weight of bipolaronic states in the bound state amounts to about $80 \%$ in the case of the $d$-wave and $50 \%$ in the case of the $p$-wave12. Thus it is apparent that in a qualitative description of current correlation function in the presence of pairing we may neglect monopolaronic states.

$s-, d$ - and $p$-wave symmetries may be realized as coherent sums of bipolaronic states 12 : $\sum_{\langle i, j\rangle} S_{\langle i, j\rangle}\left|\Psi_{\langle i, j\rangle}\right\rangle$, where $S_{\langle i, j\rangle}=1$ if $\langle i, j\rangle$ is horizontal and $S_{\langle i, j\rangle}=-1$ provided that $\langle i, j\rangle$ is vertical for the $d$-wave, while for the $p$-wave $S_{\langle i, j\rangle}$ vanishes if $\langle i, j\rangle$ is vertical, $S_{\langle i, j\rangle}=1$ provided that $j$ is on the right side of $i$ and $S_{\langle i, j\rangle}=-1$ if on the left. In order to make the story short we have skipped many details. The full construction of bipolarons that conform with irreducible representations of the point group $C_{4 v}$ may be found in an earlier article 2 .

\section{FLUX PATTERN OF THE $D$-WAVE PAIRED STATE}

As a first example of a contribution to the CCCF we consider a process shown in Fig. 1(a). The middle row and the lowest row depict string states of the type $\left|\mathcal{P}_{i}, \mathcal{P}_{j}\right\rangle$ which are coupled by action of a product of current operators on outer bonds. Circles represents positions of bipolarons, or in other words sites at which holes have been created, while thin arrows symbolize paths. In the case of the state in the second row, a single defect in the Nèel structure occupies the second site from the left, while in the case of the final state in the third row a single defect (magnon) occupies the third site from the left. Current operators which are given by the formula $j_{i j} \propto i\left(c_{i \sigma}^{\dagger} c_{j \sigma}-c_{j \sigma}^{\dagger} c_{i \sigma}\right)$ shift holes in the middle row. Holes are moved in the same direction, which is represented by the arrows in the uppermost row.

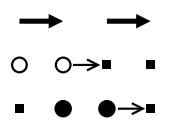

(a)

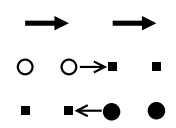

(b)

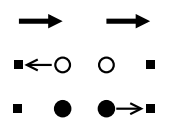

(c)

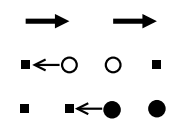

(d)
FIG. 1. Some processes which contribute to the CCCF function when holes are shifted by a product of current operators in the same direction on outer bonds.

If we adopt a convention that the direction of current on both outer bonds is from left to right the contribution to the CCCF is $t^{2} \alpha_{0,1}^{2}$. The factor $i^{2}$ which stems from product of two current operators has been absorbed by a change of sign related to the fact that the hole created at the even site in the middle row landed at a site which is occupied in the lowest row by a hole created at the odd site. Fig. 1 (a) is an example of a contribution to a the CCCF for two bonds which may be connected by a line consisting of a single bond. If we incorporate the outer bonds to that line the length of it amounts to three. Since we expect that the absolute value of amplitudes $\alpha_{\mu, \nu}$ decreases when the total length of two involved paths $\mu+\nu$ increases, only the shortest possible 
string states will decide the sign of the CCCF for two chosen bonds. Figures 1 (b), (c), (d) depict the rest of processes which involve shortest paths for holes which are moved from left to right at outer bonds of a line which length is 3 . The contributions from these processes to the CCCF are $-t^{2} \alpha_{0,1}^{2},-t^{2} \alpha_{0,1}^{2}$, and $t^{2} \alpha_{0,1}^{2}$ respectively. In the final formula these values are multiplied by factors $S_{\langle i, j\rangle}$ with which bipolaronic states at points represented by open and solid circles appear in the coherent sum that defines the bound state. Analogous processes contribute to the $\mathrm{CCCF}$ if holes are shifted from right to left on both outer bonds.

Fig. 2 depicts contributions to the CCCF for holes which move inward on outer bonds of a line of length 3.

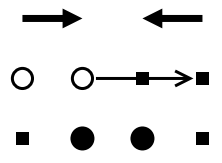

(a)

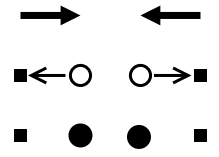

(b)

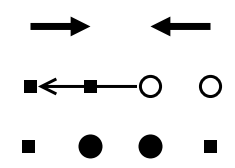

(c)
FIG. 2. Some processes which contribute to the CCCF when holes are moved by a product of current operators in opposite directions on outer bonds.

In the original string state, in the second row of Fig. 2 (a), to which the product of current operators is applied, holes occupy outer sites, while two magnons reside on a pair of sites in the middle. These magnons (defects) disappear when holes are shifted to the central position in the lowest row. Since holes that have been created in even and odd sublattices, exchange positions, the Fermi statistics again contributes the factor " -1 " but holes are moved in opposite directions, and the contribution to the CCCF becomes $-t^{2} \alpha_{0,0} \alpha_{0,2}$. Amplitudes of processes in Figures 2 (b) and (c) are $t^{2} \alpha_{0,0} \alpha_{1,1}$ and $-t^{2} \alpha_{0,0} \alpha_{0,2}$ respectively. The directions of hole motion may be inverted if the product of current operators is applied to the string state in the lowest row which gives rise to next three contributions to the CCCF on outer bonds. We can use the insights gained from the analysis of a line which consists of 3 links in total, to obtain a formula for the contribution from bipolaronic states which lie on a line of arbitrary length $l$, to the $\mathrm{CCCF}$ on outer bonds of that line,

$$
\begin{aligned}
& 2 \sum_{m=1}^{l-1} \sum_{n=2}^{l}(-1)^{m+n+1} S_{m} S_{n} t^{2} \alpha_{m-1, l-m-1} \alpha_{n-2, l-n} \\
& +2 \sum_{m=1}^{l} \sum_{n=2}^{l-1}(-1)^{m+n} S_{m} S_{n} t^{2} \alpha_{m-1, l-m} \alpha_{n-2, l-n-1},
\end{aligned}
$$

where $S_{m}$ is a coherence factor with which the bipolaron at $m$-th bond in the line appears in a coherent sum that represents a bound state. An additional lesson of the above analysis is that the shortest string states which contribute with the highest weight to the CCCF for a pair of bonds, lie on shortest paths that lead through lattice points and incorporate these bonds. If the length of such paths amounts to $l$, the length of involved strings is $l-1$, when holes are moved by a product of current operators in the same direction or $l$ and $l-2$, when the holes are shifted in opposite directions. As we have already mentioned, the leading contribution to the $\mathrm{CCCF}$ for a pair of chosen bonds is the sum of the expression (3) over all shortest paths that incorporate those bonds. Fig. 3 represents in units $10^{-5}$ the CCCF divided by the hole concentration, that has been calculated in this way for the d-wave bound state of two holes. Arrows represent the direction of current on respective bonds. We notice that despite the crudeness of the applied approximation, agreement with results of exact diagonalization performed by Leung 11 is much better than qualitative. It is an intriguing question, if that coincidence is a manifestation of a deeper relation between pairing and patterns of the CCCF. In order to see that this is the case we assume that the logarithm of the weight of a string $\alpha$ is proportional to its length $l$. That assumption is very natural because by expanding the string by one lattice spacing we raise the number of broken bonds by 2 and reach 6 longer string states, independently of the value of $l$. In our description of spin polarons parameters $\alpha_{\mu, \nu}$, where $\mu+\nu=l-1$, are related with strings of length $l$, but in general $\alpha_{\mu, \nu} \neq \alpha_{\mu^{\prime}, \nu^{\prime}}$ for $\mu+\nu=\mu^{\prime}+\nu^{\prime}$, which is the prize which we pay for pinning strings and making bipolarons localized.

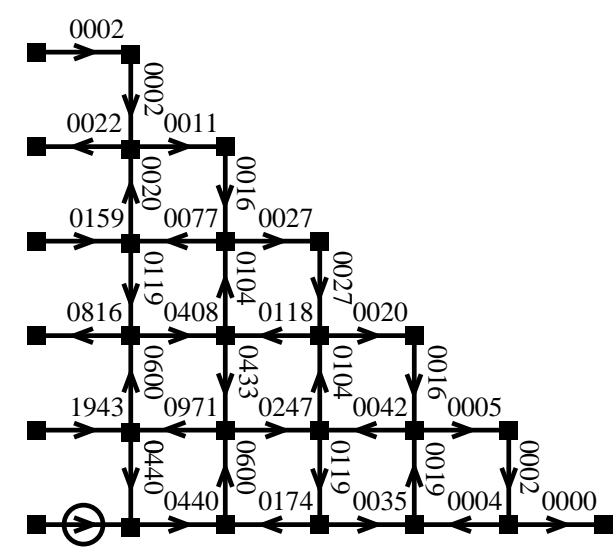

FIG. 3. $\left\langle j_{k l} j_{m n}\right\rangle / x$ in units $10^{-5}$, where $\mathrm{x}$ is the hole concentration, at $J=0.3 t$. The reference bond has been marked by a circle.

We notice that the total length of strings contributing to elements of sums in (3) is $2 l-2$. Under the previously made assumption, that $\alpha_{\mu, \nu} \propto \exp (C(\mu+\nu))$, all products of two weights $\alpha$ are the same in both sums. If we neglect them and evaluate the sum of expressions for all shortest lines that link bonds we again get the staggered flux pattern presented in Fig. A. We notice that the CCCF defined in this way has the expected structure and apart from bonds attached to the reference bond the $\mathrm{CCCF}$ is given by twice the number of shortest paths 
that connect bonds with the reference bond. This is not by coincidence, because the sum (3) with factors $\alpha$ neglected, may be evaluated explicitly,

$$
\begin{array}{r}
2 \sum_{m=1}^{l-1} \sum_{n=2}^{l}(-1)^{m+n+1} S_{m} S_{n} \\
+2 \sum_{m=1}^{l} \sum_{n=2}^{l-1}(-1)^{m+n} S_{m} S_{n}=(-1)^{l} 2 S_{1} S_{l} .
\end{array}
$$

This formula implies that the pattern of the CCCF is determined in a simple way by the symmetry of the bound state or to be more specific by the coherence factors with which bipolarons on involved bonds appear in the wave function.

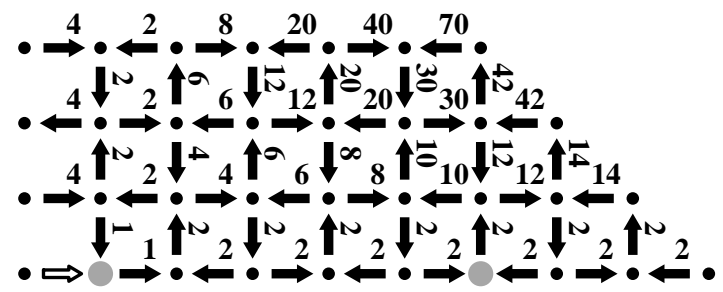

FIG. 4. The sum of expressions (3) for all shortest paths that connect bonds with a bond marked by an open arrow for the $d$-wave symmetry. The weights $\alpha$ have been neglected in the evaluation.

\section{DENSITY CORRELATION FUNCTION}

There exists another quantity which behavior is determined by existence of spin bipolarons Density matrix renormalization group calculations 20 and exact diagonalizations 11 point out that the density-density correlation function (DDCF) $C_{h h}(r)$ for holes as a function of distance $r$ between them reveals a characteristic structure in the d-wave bound state. The most pronounced feature of it is that the DDCF decays much faster along lines in the $x(y)$ direction, especially if the distance $r$ is an even number. We assume again that the highest contributions to the DDCF stem from shortest possible string states. These strings should stretch between relevant sites in order to contribute to the DDCF. Thus, the decay of the DDCF with distance is related to the decay of CCCF by the dependence of weights parameters $\alpha$ on string length. Gray disks in Fig. 4 point out to the relation between the DDCF at a pair of sites and CCCF on bonds attached to them. After a short evaluation, the contribution to the DDCF from strings that lie on a line takes the form,

$$
2 \sum_{m, n=1}^{l}(-1)^{m+n} S_{m} S_{n} \alpha_{m-1, l-m} \alpha_{n-1, l-n} .
$$

For the $d$-wave state that sum exactly vanishes for points at one of the axis $(x$ or $y$ ) if $l$ is even. Fig. 5 depicts the
DDCF obtained for the $d$-wave state by adding contributions (5) from all shortest lines that connect relevant sites.

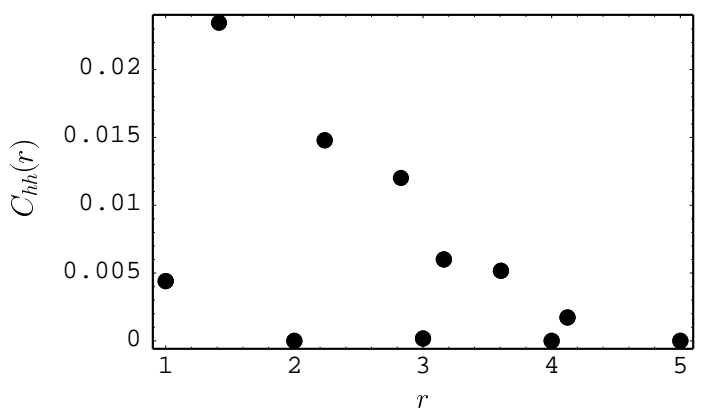

FIG. 5. Contributions to the DDCF, $C_{h h}(r)$ at $J=0.3 t$ from string states that lie on shortest paths which connect relevant sites.

Notwithstanding the simplicity of our approach the similarify with results of numerical diagonalization by Leung 11 is evident. Oscillations of $C_{h h}(r)$ with distance and minima at points which lie on an axis are visible in both calculations. Thus, we draw a conclusion that the pattern of the CCCF, the structure of the DDCF and the relation between rates of their decay with distance in the $d$-wave state is related with formation of bipolarons and strings.

\section{FLUX PATTERN OF THE $P$-WAVE PAIRED STATE}

Motivated by this observation we may try to guess the pattern of $\mathrm{CCCF}$ in the $p$-wave bound state. A state with that symmetry may became a groundstate if the exchange interaction between spins in the $t-J$ model becomes anisotropic. By applying the formula (4) we get the structure presented in Fig. 6 for the case of the symmetry $p_{x}$.

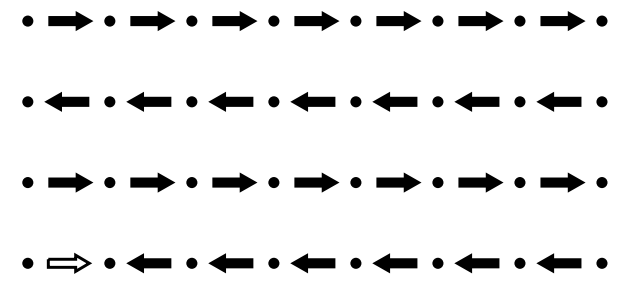

FIG. 6. The pattern of currents in the CCCF for the $p_{x}$-wave symmetry.

No current flows in the vertical direction because there are no bipolarons on vertical bonds. The cancellation which lead to the formula (4) is not exact in the case of the expression (3), and in addition some higher order contribution to the CCCF from spin bipolarons will appear for longer paths. Evaluation of these contributions 
to the CCCF is beyond the scope of this paper. Monopolarons may also obscure the picture presented in Fig. 6 because the weight of bipolarons in the $p$-wave state is smaller than for the $d$-wave state.

\section{CONCLUSIONS}

In summary, we have demonstrated that the vorticity correlations in weakly doped antiferromagnets have their origin in formation of spin bipolarons and strings. The flux pattern is determined by the symmetry of the bound state. The rate of decay with distance of different correlation functions related to current or density is determined by the rate with which the weight of strings diminishes with their length. That observation indicates that pairing and vorticity correlations in doped antiferromagnets have common origin in formation of bipolarons or in other words of strings built by magnons that connect holes at their endpoints.

The proximity of both states war already observed in mean field calculations. Conclusions drawn from that observation that pairing grows out of flux or circulating currents are questionable in the light casted by calculations presented in previous sections. Also the statement that pairs are formed by holes that circulate in opposite directions does not agree with our scenario. It seems that agreement between analytical calculations and exact diagonalizations suggest that holes that form a bound state oscillate in all possible directions at opposite endpoints of a string.

Depletion of the single particle spectral weight around the Fermi level21 known as pseudogap and exceptional non-Fermi-liquid (NFL)properties of copper-oxide based metal 22,23 are characteristic features of those systems that decide about the complexity of their physics. Some suggestions have been expressed that phase flyctuations are responsible for the pseudogap phenomenon 24. These ideas are tightly relafed with the Bose-Einstein condensation of local pair 25. The microscopic derivation in the framework of a two band model of the marginal Fermi liquid (MFL) concept26 which seems to describe quite well the NFL behavior observed in copper oxides is based on existence of circulating currents. The calculation presented in this paper suggests that both local pairs responsible for pseudogap phenomena and circulating currents which give rise to the MFL behavior may be attributed to formation of spin bipolarons. Finally, it is important to emphasize that both phenomena are tightly related and can not be discussed separately in the case of the copper oxides, which is also in agreement with the suggestion their relation with spin bipolarons and string states.

One of the authors (P.W.) acknowledges support by the Polish Science Committee (KBN) under contract No. 5 P03B 05820.
${ }^{1}$ For an early review see P.A.Lee, in High Temperature Superconductivity Proceedings, Los Alamos, 1989, ed. by K.S. Bedell et al. (Addison-Wesley, Redwood City, 1990), and also F.C. Zhang, C. Gros, T.M. Rice, and H. Shiba, Supercond. Sci. Technol. 1, 36 (1988).

${ }^{2}$ Fu Chun Zhang, Phys. Rev. Lett. 64, 974 (1990).

${ }^{3}$ Dong-Ning Sheng, Zhao-Bin Su, and Lu Yu, Phys. Rev. B 42, 8732 (1990).

${ }^{4}$ Ziqiang Wang, G. Kotliar, and Xiao-Fang Wang, Phys. Rev. B 42, 8690 (1990).

${ }^{5}$ M.U. Ubbens and P.A. Lee, Phys. Rev. B 46, 8434 (1992).

${ }^{6}$ I.S. Sandalov and M.Richter, Phys. Rev. B 50, 12855 (1994).

${ }^{7}$ E. Cappelluti and R. Zeyher, Phys. Rev. B 59, 6475 (1999).

${ }^{8}$ Xiao-Gang Wen and P.A. Lee, Phys.Rev. Lett. 76, 503 (1996).

9 P.A. Lee, N. Nagaosa, Tai-Kai Ng, and Xiao-Gang Wen, Phys.Rev. B 57, 6003 (1998).

${ }^{10}$ D. A. Ivanov, P.A. Lee, and Xiao-Gang Wen, Phys.Rev. Lett. 84, 3958 (2000).

${ }^{11}$ P.W. Leung, Phys. Rev.B 62, R6112 (2000).

12 P.Wróbel and R.Eder, Phys. Rev. B 58, 15160 (1998).

${ }^{13}$ L.N. Bulaevskii, E.L. Nagaev, and D.L. Khomskii, Sov. Phys. JETP 27F, 836 (1968).

14 B.I.Shraiman and E.D. Siggia, Phys. Rev. Lett. 60, 740 (1988).

15 R.Eder, K.W. Becker, Z. Phys. B 78, 219 (1990).

${ }^{16}$ R. Eder, Phys. Rev. B 45, 319 (1992).

17 S.A. Trugman, Phys. Rev. B 37, 1597 (1988); 41, 892 (1990).

18 P.Wróbel and R.Eder, Phys. Rev. B 49, 1233 (1994).

19 A. L. Chernyshev and P. W. Leung, Phys. Rev. B 60, 1592 (1999).

20 S.R. White and D.J. Scalapino, Phys. Rev. b 55, 6504 (1997).

${ }^{21}$ For a comprehensive review see T. Timusk and B. Statt, Rep. Prog. Phys. 62, 61 (1999), and references therein.

${ }^{22}$ H. Alloul, Phys. Rev. Lett. 63, 689 (1989).

${ }^{23}$ B. Bucher, P. Steiner, J. Karpinski, E. Kaldis, and P. Wachter, Phys. Rev. Lett. 70, 2012 (1993).

${ }^{24}$ V. Emery, and S.A. Kivelson, Nature 374, 434 (1995).

${ }^{25}$ Y.J. Uemura, Physica C 282, 194 (1997).

${ }^{26}$ C.M. Varma, Phys. Rev. b 55, 14554 (1997). 